For IEEE Nuclear Science Symposium,

San Francisco $\mathrm{Ca}$. October 29-31, 1969

RECEIVED BY DTIE MAR 20 \&970

APPROXIMATE METHODS FOR CALCULATION OF INTRINSIC

TOTAL-ABSORPTION - AND DOUBLE-ESCAPE-PEAK

EFFICIENCIES FOR Ge(Li) DETECTORS

B. A. Euler and S. N. Kaplan

October 1969

AEC Contract No. W - 7405-eng - 48
UCRL- 19392

Preprint

CONF-69101\% - ?

MTER 


\section{DISCLAIMER}

This report was prepared as an account of work sponsored by an agency of the United States Government. Neither the United States Government nor any agency Thereof, nor any of their employees, makes any warranty, express or implied, or assumes any legal liability or responsibility for the accuracy, completeness, or usefulness of any information, apparatus, product, or process disclosed, or represents that its use would not infringe privately owned rights. Reference herein to any specific commercial product, process, or service by trade name, trademark, manufacturer, or otherwise does not necessarily constitute or imply its endorsement, recommendation, or favoring by the United States Government or any agency thereof. The views and opinions of authors expressed herein do not necessarily state or reflect those of the United States Government or any agency thereof. 


\section{DISCLAIMER}

Portions of this document may be illegible in electronic image products. Images are produced from the best available original document. 
LEGAL NOTICE

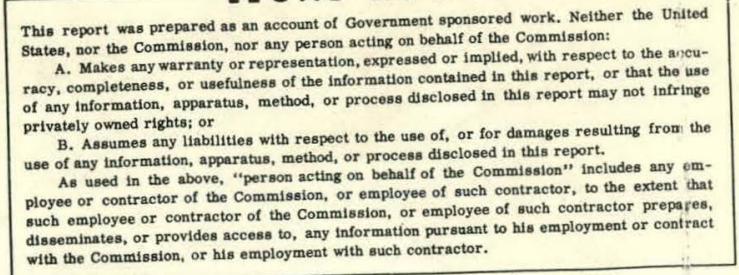

\title{
APPROXIMATE METHODS FOR CALCULATION OF INTRINSIC TOTAL-ABSORPTION-AND DOUBLE-ESCAPE-PEAK EFFICIENCIES FOR Ge(Li) DETECTORS
}

\author{
B. A. Euler* \\ Department of Nuclear Engineering \\ University of California \\ Berkeley, California \\ S. N. Kaplan \\ Department of Nuclear Engineering \\ and \\ Lawrence Radiation Laboratory ${ }^{\dagger}$ \\ University of California \\ Berkeley, California
}

\begin{abstract}
Approximate methods are presented for calculation of intrinsic total-absorption- and doubleescape-peak efficiencies of $\mathrm{Ge}(\mathrm{Li})$ detectors. These methods utilize "average" characteristics of gamma-ray photon interactions with matter, and, while developed in this paper for specific application to the $\mathrm{Ge}(\mathrm{Li})$ detector efficiency problem, should be applicable to any gamma-ray detector or absorptive medium. The technique for total-absorption-peak efficiency determination includes the use of an "average-photon energydegradation curve" and of the Dirac chord nethod for calculation of collision probabilities. The average-photon energy-degradation curve is obtained from differential Compton collision cross sections and detector material total-crosssection characteristics; it is independent of detector size and shape. The Dirac chord method is then used to determine the probability of further collisions for photons "generated" within the detector volume. In the model these probabilities are functions only of a characteristic detectors dimension, $\bar{s} \mu$, where $\bar{s}$ is the average chord length and $\mu$ is the total linear attenuation coefficient. Double-escape-peak efficiencies are determined through the calculation of the average probability of occurrence of the following three phenomena: (1) pair production,

(2) electron-positron absorption, and

(3) annihilation-photon double escape. Complete working curves and expressions are included to enable convenient utilization of these techniques. Example results are compared with efficiency determinations made experimentally and by Monte Carlo calculations.
\end{abstract}

\section{Intraduction}

The use of $\mathrm{Ge}(\mathrm{Li})$ detectors is becoming increasingly widespread, not only for nuclear spectroscopy studies, but also as an analytical tool for material analysis. In nearly all applications of the detector, knowledge of its efficiency (or relative efficiency) characteristics is essential. For detector design purposes it is also of interest to understand how a detector's efficiency is influenced by its size and shape. Since the work of Ewan and Tavendale 1 the re have been a number of reports in the literature of efficiency determinations for $\mathrm{Ge}(\mathrm{Li})$ detectors. 2

At present the experimental and the computational approaches to $\mathrm{Ge}(\mathrm{Li})$-detector-efficiency determination require considerable effort to obtain reasonably accurate results. The experimental determination requires that calibrated sources be available throughout the energy range of interest, and that the intensities of the gamma rays be known to a high degree of accuracy. Each separate detector must be individually calibrated for the entire range of energies. The Monte Carlo calculations require considerable computer time. Also, the results obtained are confined to a secific detector.

Described herein is a simplified model of the gamma-ray interaction process that permits relatively rapid and convenient hand calculations of intrinsic total-absorption- and double-escapepeak efficiencies. The simplifications introduced in the model consist in part of parameterizations of some of the functions required in the calculation, in part of averaging certain functions, and in part of making simplifying geometrical assumptions.

In the following section the calculations methods are described. Numerical values and curves are provided to allow the reader to make use of the method if he so wishes. In the next section example results a re compared with re* ported measurements and Monte Carlo calculastions. In the final section limitations and possible further applications are discussed.

\footnotetext{
* Present address: Hewlett-Packard Co., Santa Clara, California.

${ }^{\dagger}$ This work was supported in part by the U. S. Atomic Energy Commission.
} 


\section{Method}

\section{Part I - Total Absorption Peak}

Total energy absorption of an incoming gamma-ray photon occurs in a detector when there is either: (1) a photoelectric collision of the incoming photon with an electron or (2) a sequence of Compton collisions terminated by a photoelectric collision. It is assumed here that all scattered electrons are contained in the detector volume. Also, for photons less than 1.5 $\mathrm{MeV}$ in energy, pair-production effects can be neglected. The probability of an incoming photon interacting within a detector of depth $\mathrm{x}$ is

$$
P_{\text {culliaion }}=1-e^{-\mu x} \text {, }
$$

where $\mu(E)$ is the total linear attenuation coefficient. Of the collisions that do occur, $(\tau / \mu)$ a re photoelectric interactions, and $[1-(\tau / \mu)]$ are Compton interactions. These coefficients, obtained from the tabulation of Chapman, ${ }^{3}$ are shown in Fig. 1.

The Compton-scattered photons will undergo further collisions within the sensitive detector volume or escape. The scattered photons that do interact with the detector will themselves be subject to either Compton or photoelectric interactions. Each incoming photon traces a path through the detector volume which ultimately terminates in a photoelectric collision or an escape. The total-absorption probability can be represented by the expression

$$
\begin{aligned}
& \text { Total-absorption probability }=\mathrm{P}_{0}+\mathrm{C}_{0} \mathrm{P}_{1} \\
& +\mathrm{C}_{0} \mathrm{C}_{1} \mathrm{P}_{2}+\cdots+\mathrm{C}_{0} \mathrm{C}_{1} \mathrm{C}_{2} \cdots \mathrm{C}_{n-1} \mathrm{P}_{n}+\cdots
\end{aligned}
$$

where $\mathrm{P}_{0}=$ probability that the incoming photon, of energy $E_{0}$, undergoes photoelecLic cullision,

$C_{0}=$ probability that the incoming photon undergoes Compton collision,

$\mathrm{P}_{1}=$ probability that the singly-Comptonscattered photon of reduced energy $\mathrm{E}_{1}$ has a photoelectric collision,

$\mathrm{C}_{n-1}=$ probability that an $(n-1)$ th-scattered photon of reduced energy $E_{1}$ has a Compton collision,

$P_{n}=$ probability that an $(n)$ th-scattered photon of reduced energy $E_{n}$ has a phntenelectric collision.

The basis of this calculation is to treat each te $\mathrm{rm}$ in the above expression as the product of independent average probabilities. The first probabilities of the collision chain are, from (1),

$$
P_{0}=(\tau / \mu)\left(1-e^{-\mu x}\right) \text { and } C_{0}=(1-\tau / \mu)\left(1-e^{-\mu x}\right),
$$

where $x$ is the detector depth. For the

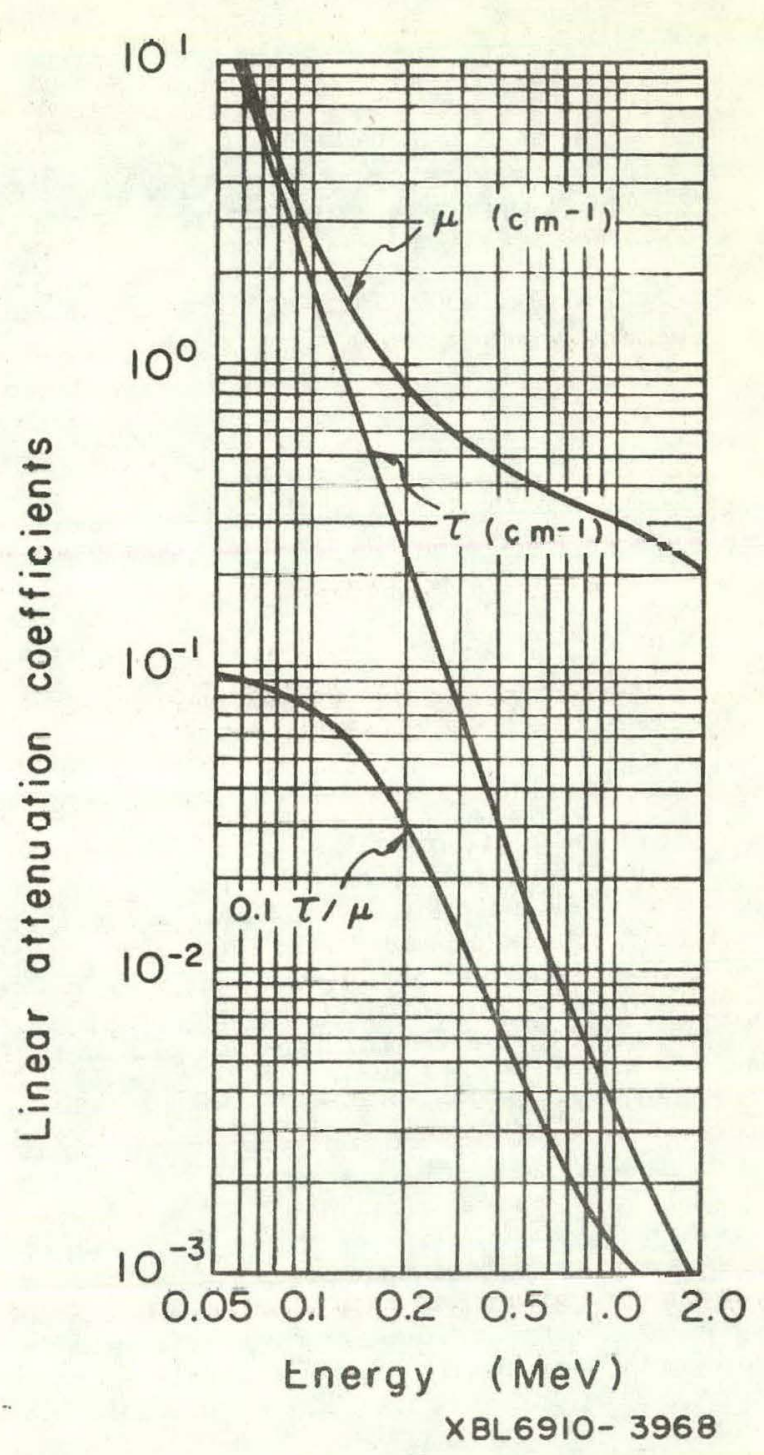

Fig. 1. Germanium linear attenuation cocfficients, showing the total, $(\mu)$, photoelectric only, $(\tau)$, and the ratio of photoelectric to total $(\tau / \mu)$. (From Chapman, Ref. 3).

remainder of the $\mathrm{P}$ and $\mathrm{C}$ probability determinations anuther approach must be utilized.

The average cross section for a second collision is determined for all Compton-scattered photons resulting from the initial collision of a particular-energy gamma ray, $E_{0}$. It can be oblained from the following expression:

$$
\bar{\sigma}_{t}\left(E_{0}\right)=\frac{\int_{0}^{\pi} \sigma_{t}\left[E\left(\theta, E_{0}\right)\right] \frac{d \sigma}{d \theta}\left(E_{0}\right) d \theta}{\sigma_{c}{ }^{\left(E_{0}\right)}} \text {, }
$$

where $\bar{\sigma}_{t}=$ average total cross section seen by a Compton-scattered photon.

$\sigma_{t}=$ total cross section seen by photons of reduced energy $E$ after being scattered into angle $\theta$,

$\frac{\mathrm{d} \sigma}{\mathrm{d} \theta}=$ differential Compton cross section 


$$
\begin{aligned}
& \text { for incident photons of energy } \mathbf{E}_{0} \text {. } \\
& \sigma_{c}=\text { total Compton cross section. }
\end{aligned}
$$

Numerical integrations were performed for a number of incident photon energies, using the cross-section values of Nelms. ${ }^{4} \mathrm{As}$ each $\sigma_{t}$ is unique to a particular energy photon, the $\bar{\sigma}^{\prime}$ 's may be used to define the energy of an "average" degraded photon. A plot of average-degraded-photon energy as a function of incident photon energy is given in Fig. 2. The once-scatte red photon obtained from this plot can the, in turn, be considered to the incident photon for another collision for which a twice-scattered average-photon energy can be determined. An "average-photon energydegradation chain" can therefore be establisked with this curve for any initial gamma-ray photon energy up to $2 \mathrm{MeV}$.

For the calculation of subsequent collision probabilities we assume each Compton-scattered photon to have a characteristics "average" energy as determined above and, on the average, to originate uniformly throughout the detector volume with an isotropic angular distribution.

The calculation of the probability of a collision, or successive collisions, by averaging methods has received considerable attention in nuclear reactor analysis, particularly with regard to fast-neutron collisions in fuel elements. We have adopted such a method here, the chord method, first used by Dirac. 5 We use the escape probability curves of Dresner, given as a function of a "characteristic dimension!' $\bar{s} \mu$, where $\bar{s}$ is the average chord length of a volume and $\mu$ is the linear attenuation coefficient. The quantity, $\bar{s}$, for a volume with a nonreentrant surface, has the simple form

$$
\overline{\mathrm{s}}=4 \mathrm{~V} / \mathrm{A} \text {, }
$$

where $\mathrm{V}$ is the volume and $\mathrm{A}$ is the total surface area of the body. Thus, the "characteristic dimension, " $\bar{s} \mu$, can be determined readily for a specific detector and photon energy. The results of Dresner are given in Fig. 3 for a sphere, an infinite cylinder, and an infinite slab. For the results reported here the sphere data were used. 7

The steps, then for determining each of the $P_{n}$ and $C_{n}$ values of Eq. (2) are as follows:

1) The energy, $E_{n}$, of the average nth scattered photon is found from $E_{n-1}$ and Fig. 2 (where $E_{0}$ is the energy of the incident photon).

2) For the photon of energy $E_{n}, \mu$ and $(\tau / \mu)$ are then obtained from Fig. 1.

3) The interaction probability, $P_{C}$, is read as a function of $\bar{s}_{\mu}$ from Fig. 3(using the curve labeled "sphere"), where $\bar{s}$ was calculated from Eq. (5).

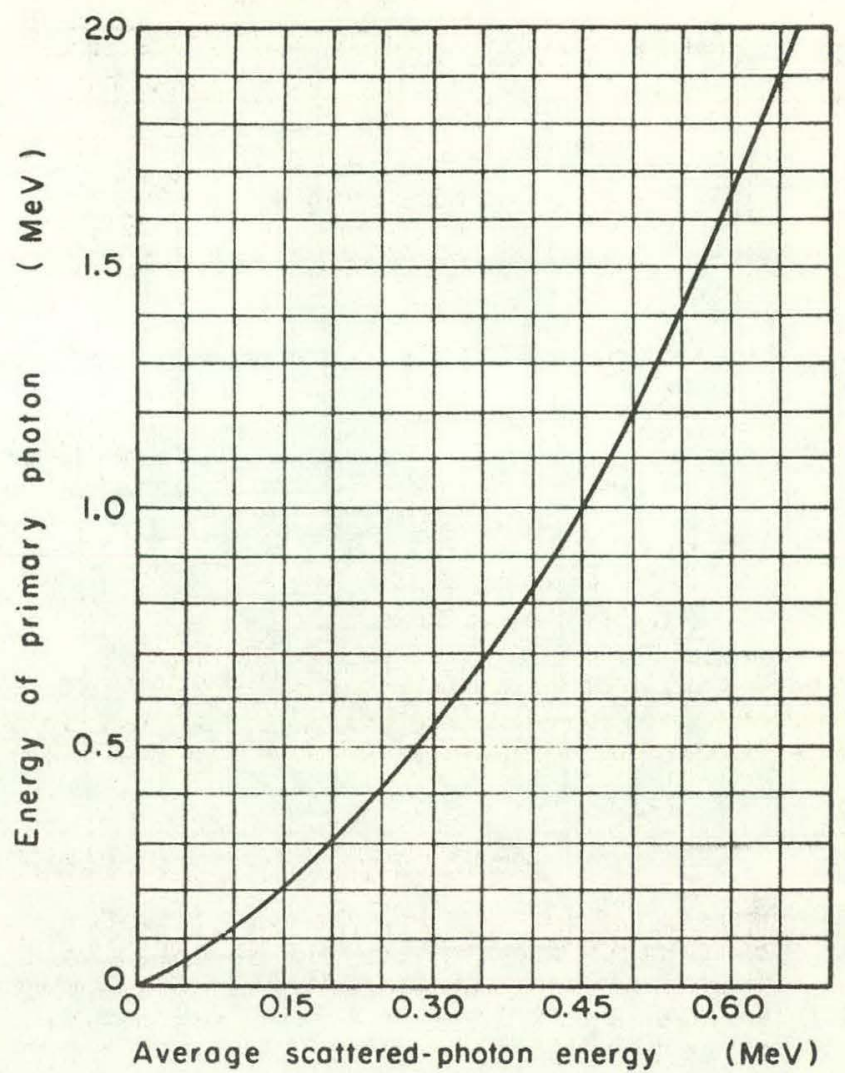

XBL 6910-3969

Fig. 2. Average-energy-degradation curve for $\mathrm{Ge}$.

4) Then,

$$
\begin{aligned}
& P_{n}=(\tau / \mu) P_{c^{\prime}} \\
& C_{n}=[1-(\tau / \mu)] P_{c} .
\end{aligned}
$$

Finally, the $P_{n}$ and $C_{n}$ values are combined in Eq. (2) to give the total absorption probability. The sum [Eq. (2)] typically converges to within $1 \%$ of its total value in a maximum of $\mathrm{six}$ to seven terms.

\section{Part II - Double-Escape Peak}

Double-escape-peak efficiencies are calculated as the product of the probabilities of (a) pair production, (b) total absorption of the electron-positron pair, and (c) complete escape of the positron-annihilation gamma rays. It is assumed in the calculation that these three probabilities can be treated independently.

Thus,

$$
\mathrm{P}_{\text {dep }}=\mathrm{P}_{\mathrm{pp}} \cdot \mathrm{P}_{\mathrm{A}} \cdot \mathrm{P}_{\mathrm{de}}
$$

where $\mathrm{P}_{\text {dep }}$ = probability of a double-escape-peak

$$
P_{p p}=\text { probability of pair production, }
$$




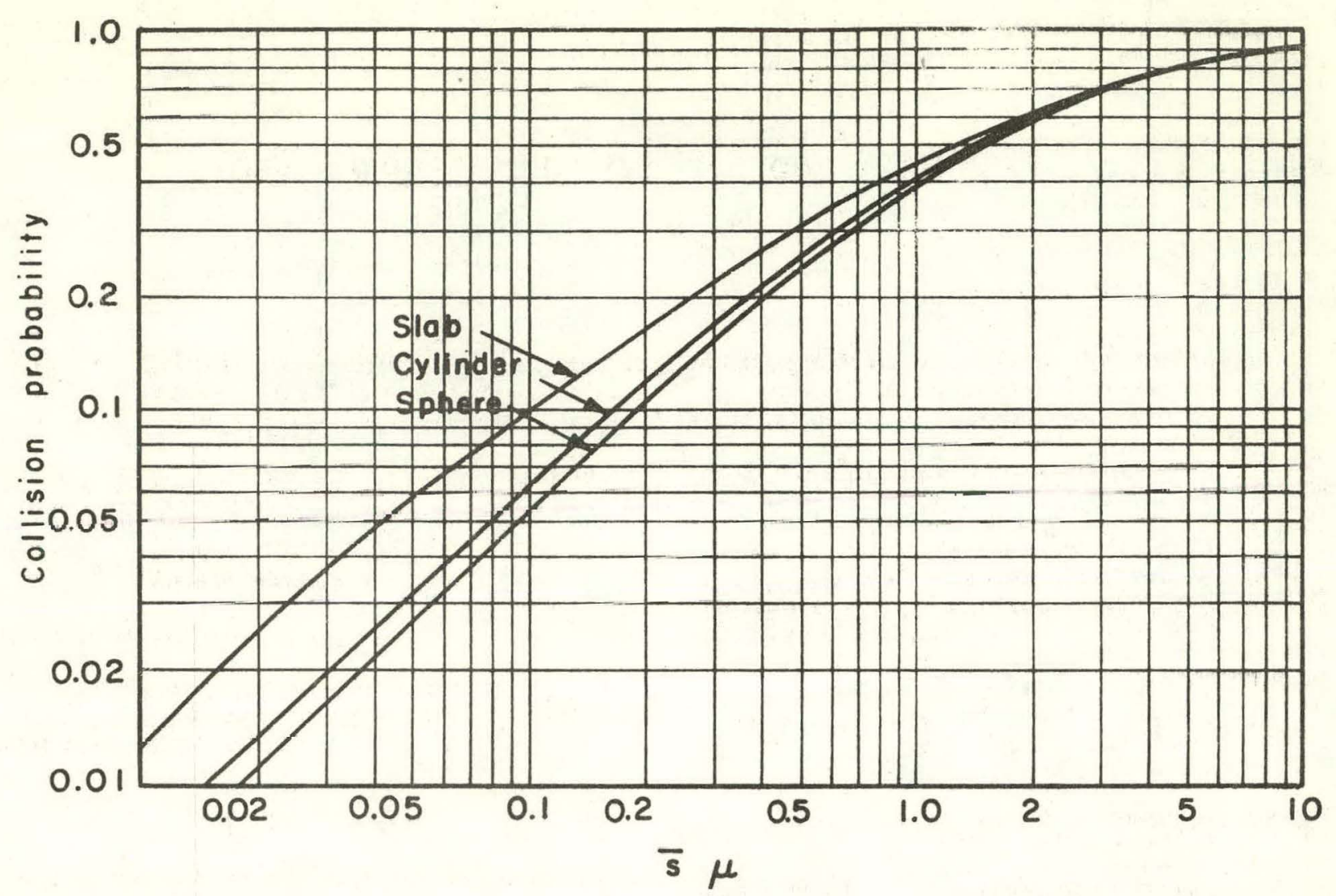

XBL6910 3971

Fig. 3. Collision probability as a function of the characteristic dimension, $\bar{s} \mu$, for a sphere, an infinite cylinder, and an infinite slab. The paranteler $\mathbf{s}$ is the avesdge cord length of a volume and $\mu$ is the total linear attenuation coefficient.

$P_{A}=\begin{aligned} & \text { prnbability for eleclrun-positron } \\ & \text { absorption, }\end{aligned}$

$P_{\text {de }}=$ probability of annihilation photon double escape.

\section{(a) Pdir Production}

The probability for pair production, $P_{p p}$, in a detector of thickness $x$ is given by $\mathrm{Eq}$. ( 19 , multiplied by $\kappa / \mu$

$$
P_{P p}=\frac{\kappa}{\mu}\left(1-e^{-\mu x}\right) \text {. }
$$

where $k$ is the partial at.esuation coefficient for a pair-productios interaction. The linear attenuation coefficientis for germanium in the energy range of interest here are given in Fig. 4.

\section{(b) Electron-Positron Absorption}

Whereas the probability of electron escape was neglected in Part I, here, because of the higher electron energies, it is a much more important factor and must be considered in some detail. It is, however, assumed that the electrons are emitted in the forward direction. This sim- plifies the problem to one-dimensional geometry.

F'or a photon of energy $E_{y}$, the total energy available to the electron-positron pair is $\mathrm{E}_{\mathrm{p}}$ $=E_{\gamma}-1.022 \mathrm{MeV}$. If the higher energy of the twon electsuis is $E_{1}$ then the probability of total absurption of the pair is

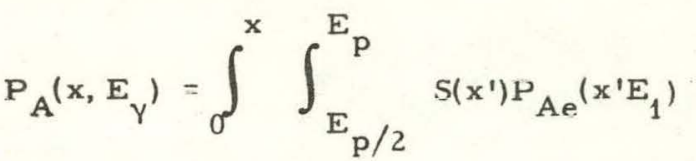

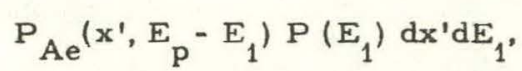

where $S\left(x^{\prime}\right)$

$=$ relative probability of production of particle pairs at $\mathbf{x}^{\prime}$, $\mathrm{P}_{\mathrm{Ae}}\left(\mathrm{x}^{\prime}, \mathrm{E}\right)={\text { absorption probabilitiy of } \mathrm{e}^{-}}^{\left(\text {or } \mathrm{e}^{+}\right) \text {produced at } \mathrm{x}^{\prime} \text { with }}$ energy $E$,

$P\left(E_{1}\right) \quad=$ probability of the higher energy particle having energy $E_{1}$,

$P_{A}\left(x, E_{\gamma}\right)=$ probability of two-particle absorption in detector of width $x$. 


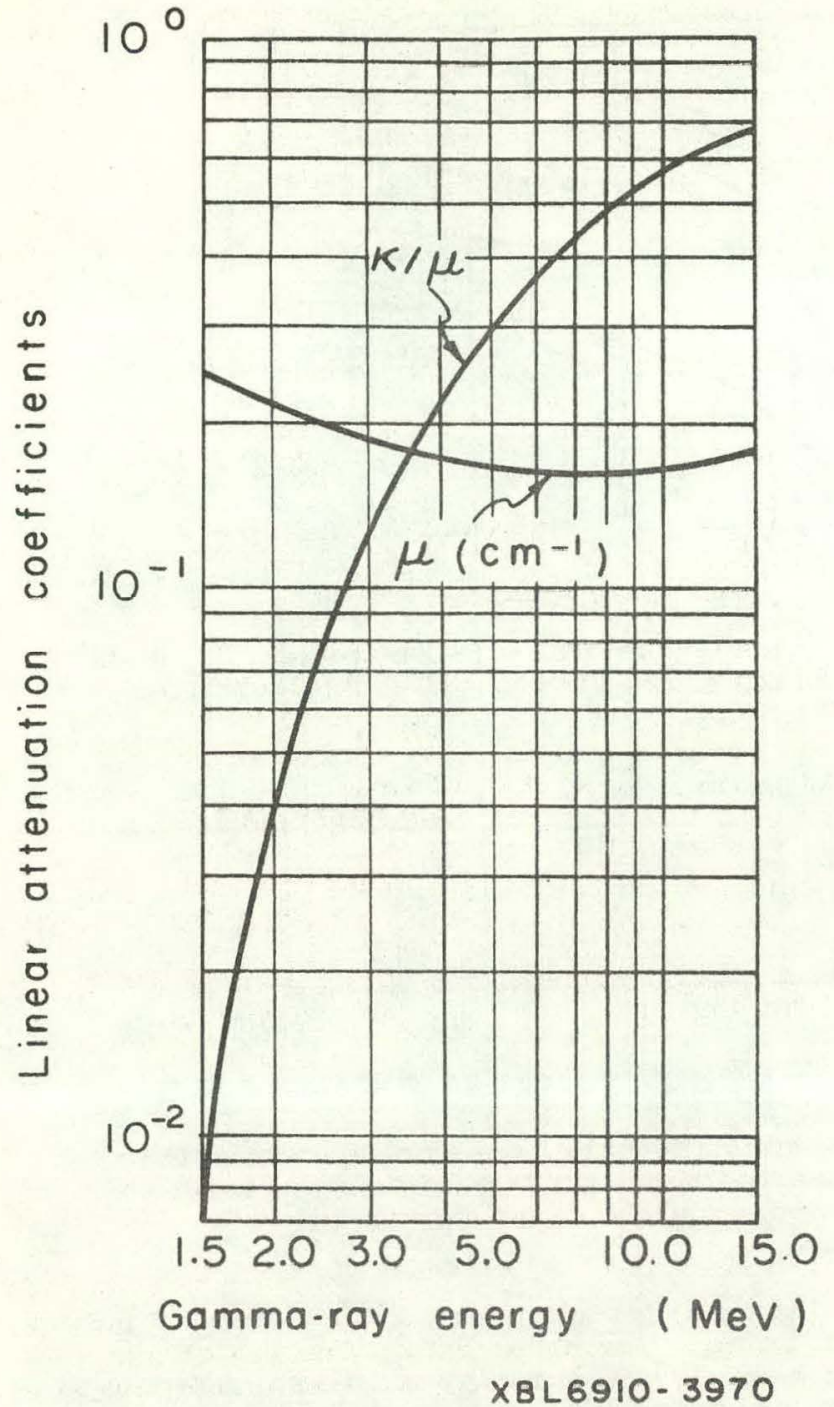

Fig. 4. Germanium linear attenuation coefficients. The curves shown are the total attenuation coefficient $(\mu)$ and the ratio of pair-production interactions to total $(\kappa / \mu)$. (From Chapman, Ref. 3).

In the evaluation of this expression the following simplifying approximations arc made: ${ }^{2}$

1) Attenuation of the incident gamma-ray beam is neglected. Hence

$$
S\left(x^{\prime}\right) \equiv 1 / x \text {. }
$$

2) All values of $e^{-}\left(e^{+}\right)$energy are assumed equally likely. Hence

$$
P\left(E_{1}\right) \equiv 2 / E \text {. }
$$

3) The probability of electron containment, $\mathrm{PA}_{\mathrm{A}}\left(\mathrm{x}^{\prime}, E\right)$, was parameterized by using as a guide the electron absorption data of Marshall and $\mathrm{Ward}^{8}$ and the range relationship given in Siegbahn. The simplified forms used were

$$
\begin{array}{rlrl}
P_{A}\left(x^{\prime}, E\right) & =0 & & x^{\prime}<0.24 R \\
& =\left(x^{\prime}-0.24 R\right) /(0.76 R) & 0.24 R \leqslant x^{\prime} \leqslant R,
\end{array}
$$

where $\mathrm{R}$, the maximum electron range, was taken to be

$$
R=0.1087 \mathrm{E}(\mathrm{MeV})
$$

The calculated $P_{A}\left(x, E_{\gamma}\right)$ is shown in Fig. 5 (top curve) as a function of $x / R_{M}$, where $R_{M}$ is $R\left(E_{\gamma}-1.022 \mathrm{MeV}\right)$.

In addition, bremsstrahlung effects were considered. At these energies there is a significant probability that electrons emit bremsstrahlung and that the bremsstrahlung escape.

To parameterize this effect it was assumed that the probability per unit path length of emission of a bremsstrahlung of energy $E_{B}$ was $P_{B}$ $=\mathrm{a} / \mathrm{E}_{\mathrm{B}}$, where $\mathrm{a}$ is a constant.

The probability per unit path length that an electron of energy $E^{\prime}$ will produce a bremsstrahlung of energy greater than $\epsilon$ then becomes

$$
\int_{\epsilon}^{E^{\prime}} a / E_{B} d E_{B}=a \ln E^{\prime} / \epsilon \text {. }
$$

If $P_{\bar{B}}(y)$ is defined as the probability that an electron travels a distance y without producting a brems strahlung of energy greater than $\epsilon$ and it is assumed that the total path length traveled by an electron of energy $\mathrm{E}$ is its maximum range, $\mathrm{R}$, then

$$
P_{\bar{B}}(R)=\exp \left[-\left(a R_{\epsilon} / E\right)(E / \epsilon \ln E / \epsilon-E / \epsilon+1)\right] .
$$

For the results presented here $\epsilon$ has been set equal to $0.1 \mathrm{MeV}$. This means that, for the purposes of these calculations, all bremsstrahlung of lower energy are assumed to be contained by the crystal and all of higher energy are assumed to escape ( $L$. Fig. 1). The constant, $a=\ell_{R}$ where $\ell R$ is the radiation length in $G e$, was taken to be $a=0.423 \mathrm{~cm}^{-1} .10$ When the factors $P \bar{B}\left[R\left(E_{1}\right)\right]$ and $P \bar{B}\left[R\left(E_{p}-E_{1}\right)\right][E q .(9)]$ are included inside the intergal [Eq. (8)] we obtain, by numerical integration, a $\mathrm{P}_{A}\left(\mathrm{x}, \mathrm{E}_{\gamma}\right)$ that is explicitly energy dependent. A family of curves for $E_{Y}=2,4,6,8$, and $10 \mathrm{MeV}$ is shown in Fig. 5.

\section{(c) Annihilation-Photon Double Escape}

The probability of annihilation-photon double escape, $P_{\text {de }}$, is obtained by using Fig. 3. Whereas previously the parameter of interest was the probability of collision, now the parameter of interest is the probability of complete photon escape from the detector volume. The procedure, then, is to determine the characteristic dimension $\bar{s}_{\mu}$ for the $0.511-\mathrm{MeV}$ photon for the detector studied. With the characteristic dimension, the probability of collision is determined ("sphere" curve). The probability of escape, Pesc' is 


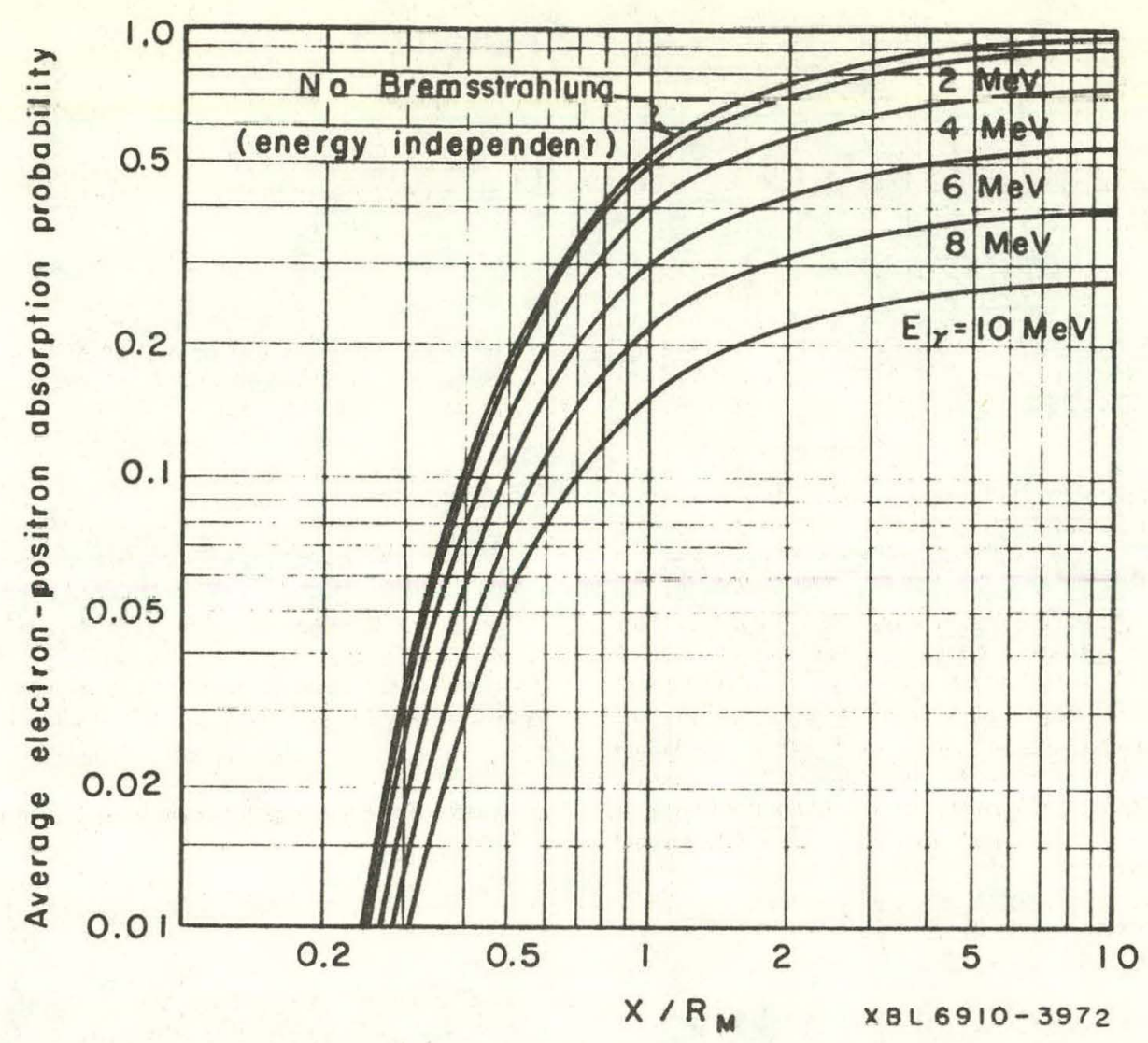

Fig. 5. Average electron-positron energy absorption probabilities as a function of the ratio of detector depth to maximum electron range $\left(x / R_{M}\right)$. In the top curve the effect of bremsstrahlung is not included and the results are energy independent.

$$
P_{\text {esc }}=1-P_{c} \text {. }
$$

The probability of double escape then is

$$
P_{\text {de }}=P_{\text {esc }}{ }^{2}=\left(1-P_{c}\right)^{2} \text {. }
$$

To review: the steps for determining the doubleescape-peak etticiency for a gamma ray of energy $\mathrm{E}_{Y}$ are:

1) For $E_{\gamma}$, find $\mu$ and $\kappa / \mu$ (Fig. 4) and calculate $\mathrm{P}_{\mathrm{pp}}[\mathrm{Eq} .(7)]$.

2) Evaluate $x / R_{M}=x /\left[0.1087\left(E_{\gamma}-1.022\right)\right]$ and obtain $P_{A}$ from Fig. 5 .

3) Evaluate $\bar{s} \mu\left(\mu_{0}, 511 \mathrm{MeV}=0.42 \mathrm{~cm}^{-1}\right)$. obtain $P_{S}$ from Fig. 3 , and evaluate $P_{d e}$ $=\left(1-P_{c}\right)^{2}$.

4) The efficiency is then determined as the product of the above three results $[\mathrm{Eq} .(6)]$.

\section{Results}

Figure 6 presents a comparison of the intrinsic total-absorption-peak-efficiency values of two planar $\mathrm{Ge}(\mathrm{Li})$ detectors of the same reported cross section area $\left(2.5 . \mathrm{cm}^{2}\right)$ but of olightly different iepuiled thickness $(3.5 \mathrm{~mm}$ vs. $4.0 \mathrm{~mm}$ ). The upper data points (Ewan and Tavendale ${ }^{1}$ ) and the lower experimental curve of Cline, 11 (त), a re rnmpared with (a) Monte Carlo calculations of Wainio and Knoll, 12 (b) Monte Carlo calculations of de Castro Faria and Levesque, 13 and (c) results of this paper. In both cases the curve shapes calculated by the method given here agree quite well with experiment. Although the Monte Carlo calculations fit the upper data more closely, ours fit more closely to the lower, both in value and in shape. In both cases the differences hetween the efficiences calculated here and the experimental measurements are less than, or un the order of, 0.5 inn delectur-thickness equivalent.

In Figure 7 calculations are compared with reported measurements on three larger detectors. The upper set of data (d) shows the experimental curve of Cline 11 (the reported area of his detector was actually $4.9 \mathrm{~cm}^{2}$ ) and two data points of Ewan and Tavendale. 1 The lower set of data points is from Michaelis. 14 The authorship of the calculated curves is indicated by the same letter symbols used in Fig. 6. The $X^{\prime}$ s on curve (a) show the energy values at which the Monte Carlo calculations were made. Again the curve shapes calculated here agree quite well with 


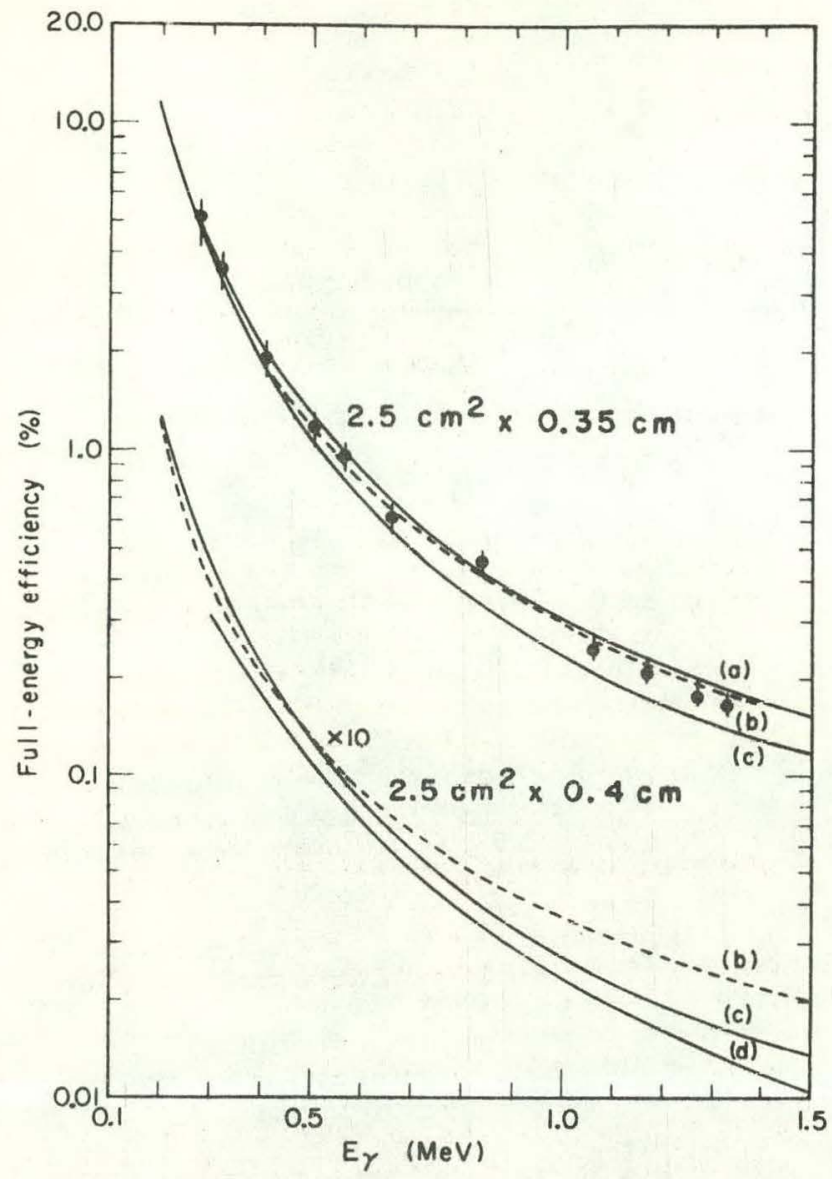

X8L6910-6038

Fig. 6. Total-absorption-peak efficiencies for two detectors, each of crosssection area $2.5 \mathrm{~cm}^{2}$. The experimental measurements of Ewan and Tavendale (Ref. 1) a re shown as data points. The curves represent the following.

(a) the Monte Carlo calculations of Wainio and Knoll (Ref. 12).

(b) the Monte Carlo calculations of de Castro Faria and Levesque (Ref. 13).

(c) the present calculations.

(d) the experimental measurements of Cline (Ref. 11).

experiment; however, the absolute values calculated are in somewhat greater disagreement than for the smaller detectors (Fig. 6). It should be pointed out, though, that in the upper set of data the reported experimental results for two detectors of the same thickness and cross-section area differ from each other by an even larger amount, and the ralculated curve of this work lies between them.

Figure 8 preocnto a comparison of inlrinsic double-escape-peak-efficiency values of a $2.5 \mathrm{~cm}^{2}$ $\times 0.35 \mathrm{~cm}$ planar $\mathrm{Ge}(\mathrm{Li})$ detector. The data points are those of Wean and Tavendale. 1 Curves (a) and (b) are the Monte Carlo-calculated results of

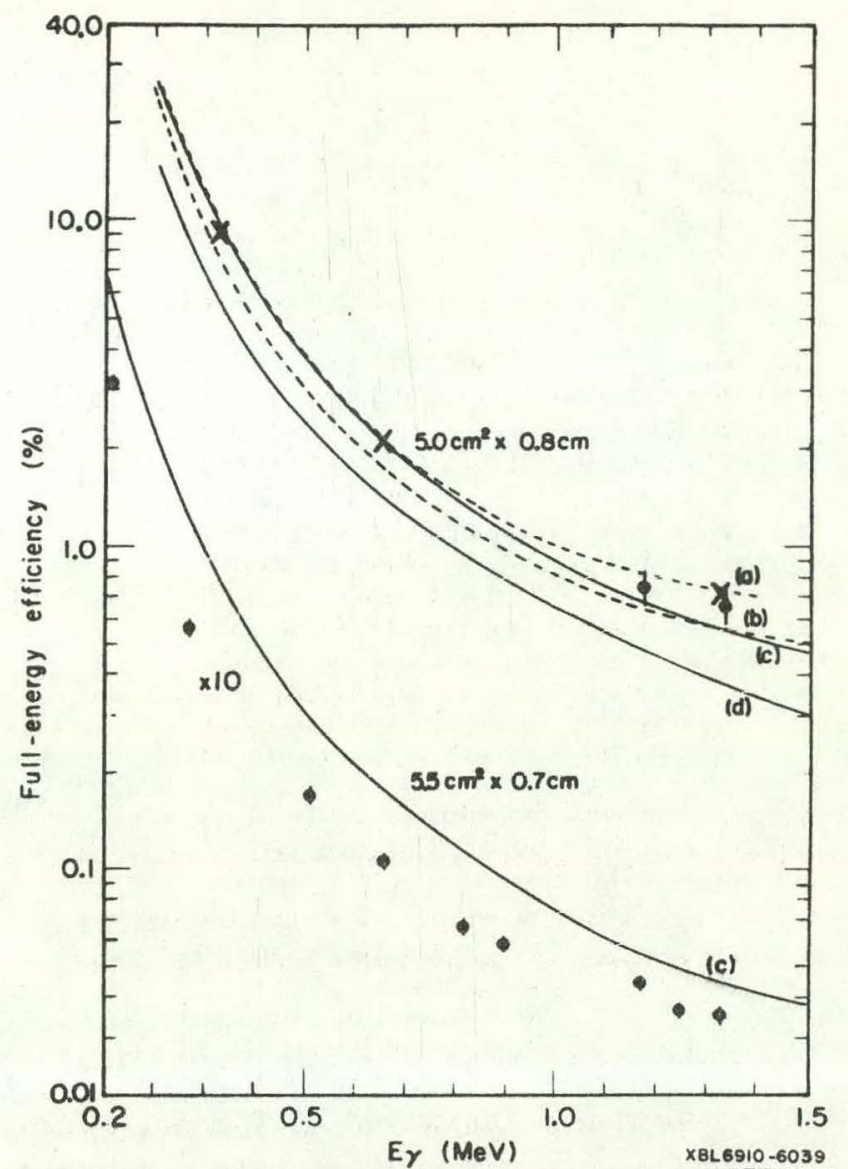

Fig. 7. Total-absorption-peak efficiencies for two detectors. The experimental measurements of Ewan and Tavendale (Ref. 1) are shown as data points in the upper group of curves, while the measurements of Michaelis (Ref. 14) are shown beneath the lower curve. The Monte Carlo-calculated efficiency values of Wainio and Knoll (Ref. 12) are indicated by crosses, $x$. The curves represent the following:

(a) the Monte Carlo calculation of Wainio and Knoll (Ref. 12).

(b) the Monte-Carlo calculations of de Castro Faria and Levesque (Ref. 13).

(c) the present calculations

(d) the experimental measurements of Cline (Ref. 11).

de Castro Faria and Levesque ${ }^{13}$ and Wainio and Knoll, 12 respectively. Curve (c) presents the results obtained by the method of this work.

Figure 9 presents further comparisons of the results of this work [curve (b)] with the calculated results of de Castro Faria and Levesque 13 and the experimental-measurement results of Cline. 11 Monte Carlo-calculated data points of Wainio and knoll 12 are indicated as circles, $U$.

The results of the method of this work more closely resemble the Monte Carlo-calculated results of Wainio and Knoll ${ }^{12}$ (this agreement is 


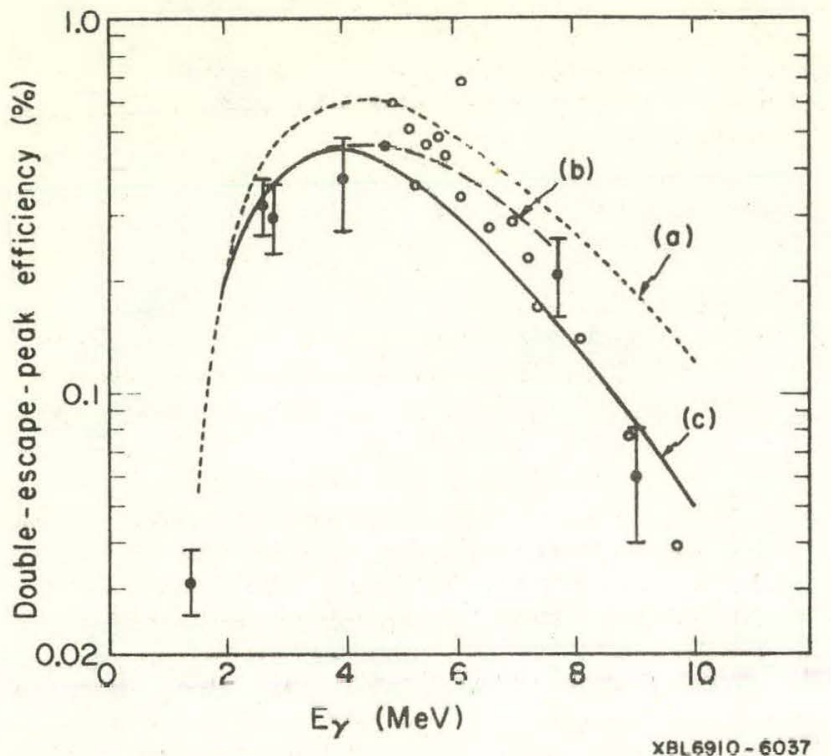

Fig. 8. Double-escape-peak efficiencies for a $2.5 \mathrm{~cm}^{2} \times 0.35 \mathrm{~cm}$ detector. The experimental measurements of Ewan and Tavendale (Ref. 1) are shown as data points. The curves represent the following:

(a) the Monte Carlo calculations of de Castro Faria and Levesque (Ref. 13).

(b) the Monte Carlo calculations of Wainio and Knoll (Ref, 12).

(c) the present calculations.

remarkable in Fig. 9), who considered bremsstrahlung cffecls in lieir calculation, than the results of de Castro Faria and Levesque, 13 who did not. The present hand-calculation technique gave results very close to the measured values of Ewan and Tavendale, ${ }^{1}$ but higher than the measured values of Cline ${ }^{11}$ for a pair of detectors not ton different in oize. In order to ease the calculational effort in the further application of this method to testing the ettects of various parameter adjustments, a computer program has been written that will calculate and plot efficiencies in the manner described here. The resulis for a sample tamily of detectors are shown in Figs. 10, 11, and 12. Figures 11 and 12 show double-escape results with and without consideration of bremsstrahlung effects.

\section{Discussions and Conclusions}

The semianalytical approximation method described here produces results in good agreement both with Monte Carlo calculations and experiment. Differences from Monte Carlo calculations are comparable to the differences between two Monte Carlo calculations. Furthermore, the method reliably reproduces the shape of measured $\mathrm{Ge}(\mathrm{Li})$ efficiency curves. Where differences exist between calculation and measurement they are almost proportional over the entire range of energies considered.

Absolute-value disagreements with experiment may be related to certain approximations. How-

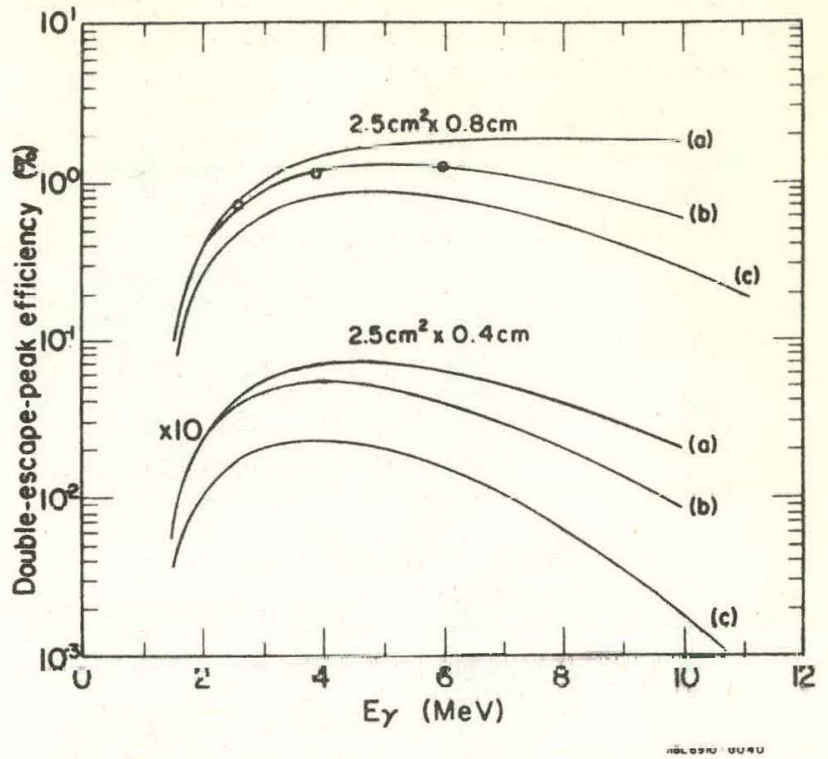

Fig. 9. Double-escape-peak efficiencies for two detectors. The Monte Carlo calculations of Wainio and Knoll (Ref. 12). for a $2.5 \mathrm{~cm}^{2} \times 0.8 \mathrm{~cm}$ detector are shown as rircles, $O$. The curves represent the following:

(a) the Monte Carlo calculations of de Castro Faria and Levesque (Ref. 13).

(b) the present calculations.

(c) the experimental measurements of Cline, (Ref. 11).

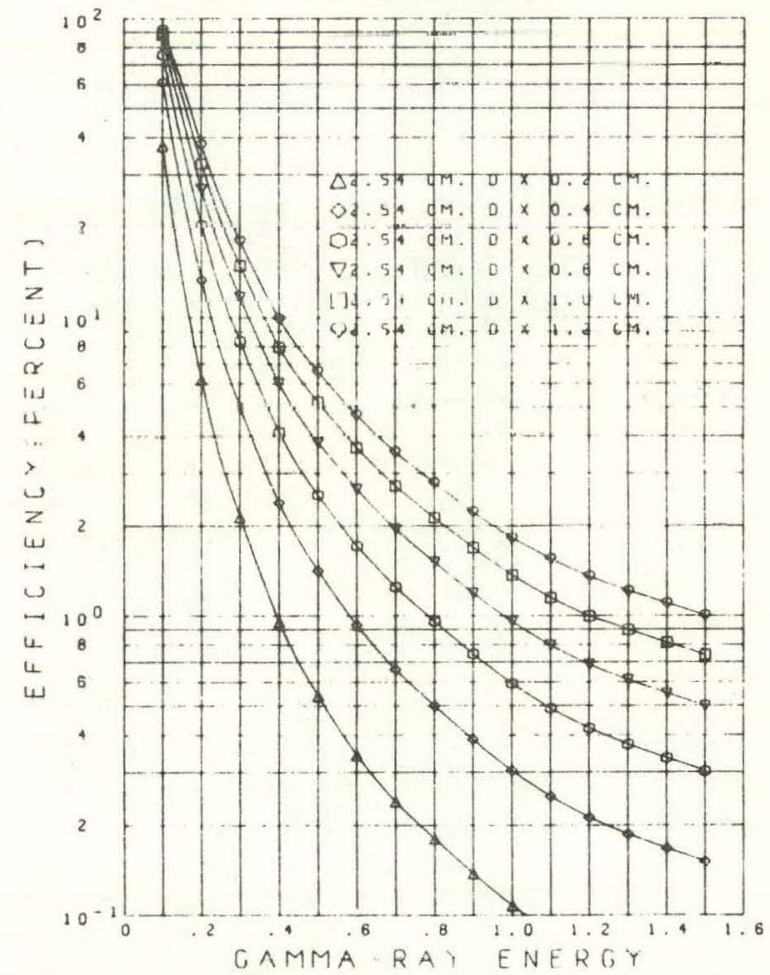

XHL 6910-585

Fig. 10. Calculated $\mathrm{Ge}(\mathrm{Li})$ intrinsic full-energypeak efficiencies for a family of cylindrical planar detectors. 


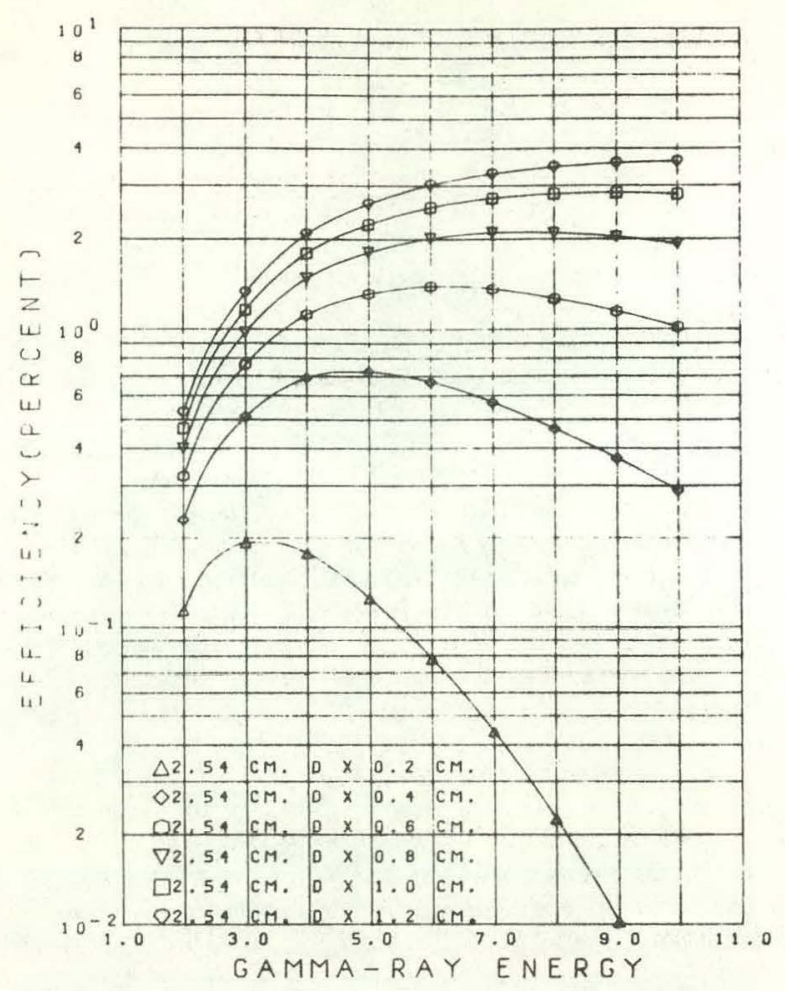

XBL 6910-5854

Fig. 11. Calculated $\mathrm{Ge}(\mathrm{Li})$ intrinsic doubleescape-peak efficiencies for a family of cylindrical planar detectors. Losses due to bremsstrahlung are neglected.

ever, on the average the Monte Carlo disagreement with experiment is even greater. Other possible sources of this disagreement lie in the interpretation of experimental data. The calculation assumes a parallel beam uniformly illumi nating the front surface of the detector and that the detector is sensitive at its surface and over the entire reported volume. Nonparallel incidence, a surface dead layer, and a somewhat reduced sensitive volume can each contribute to disagreement between experiment and calrulation. [A. slight correction was included here for the 0.5 $\mathrm{mm}$ dead layer reported by Michaelis (Fig. 7); the correction was observable only at energies below $0.2 \mathrm{MeV}]$. The true sensitive dimensions of a detector are not easily determined. This question is considered in some detail by Walford and Doust. 15

At present the method is applicable only to materials with nonreentrant surfaces. Therefore, excluded from the present analysis are five-sided and coaxial detectors. However, it is hoped that with some modification the method will eventually lend itself to this analysis also. In addition, it should be pointed out that for detectors with dimensions comparable to gamma-ray collision mean free paths, one of the assumptions, that of uniform volume distribution of secondary sources, is no longer valid. Hence, calculations using the methods described here are expected to be less applicable for very large detectors.

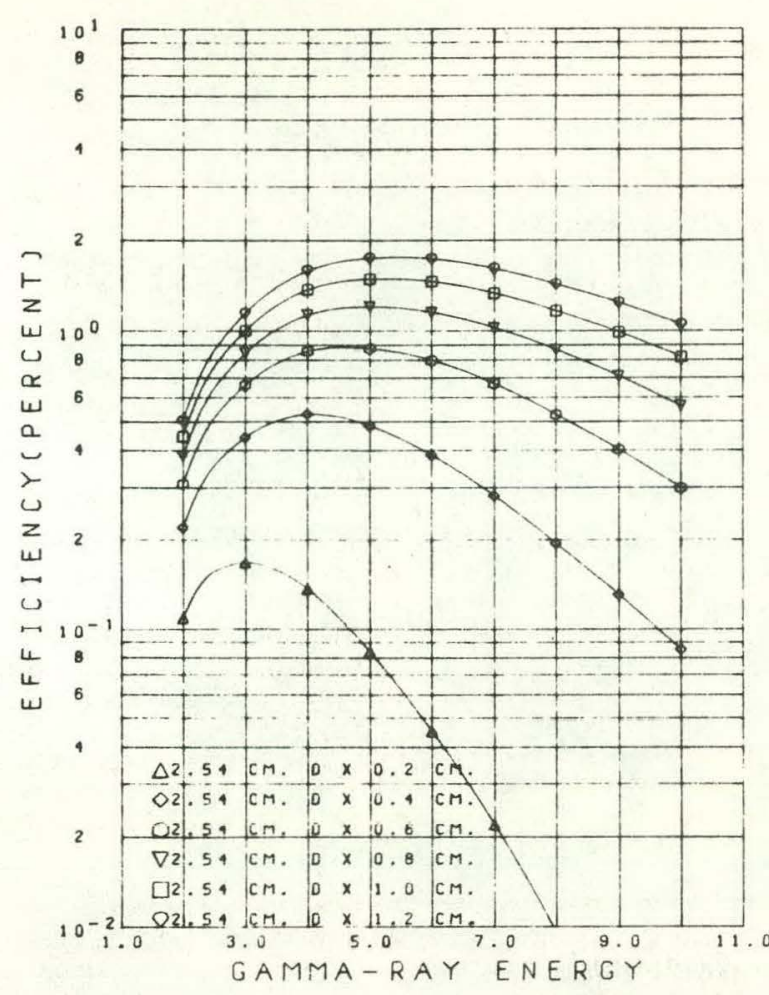

XBL 6910-5852

Fig. 12. Calculated $\mathrm{Ge}(\mathrm{Li})$ intrinsic doubleescape efficiencies for a family of cylindrical planar detectors. Losses due to bremsstrahlung are included.

In summary, the methods developed here should considerably ease the fairly precise determination of theoretical efficiencies for detectors of various sizes and shapes. It is further hoped that the use of the technique will lead to greater qualitative understanding of the relative importance of various aspects of photon-energy containment by gamma-ray detectors. The Dirac chord method should prove especially useful for photon detection and attenuation problems.

\section{Acknowledgments}

The authors would like to express their gratitude to $\mathrm{Mr}$. Robert New who was instrumental in adapting these calculations to computer processing. We would also like to thank $\mathrm{Mr}$. Gary Born who performed the bremsstrahlung calculations.

\section{REFERENCES}

1. G. T. Ewan and A. J. Tavendale, Can, J. Phys. 42, 2286 (1964).

2. For a detailed bibliography, see B. Euler, M.S. thesis, University of California, Berkeley, 1969. Also presented here is a more detailed description of the development of most of the approximations used in these calculations.

3. G. T. Chapman, Nucl. Instr. Methods $\underline{52}$, 101 (1967).

4. A. T. Nelms, Graphs of the Compton Energy- 
Angle Relationship and the Klein-Nishina Formula from $10 \mathrm{keV}$ to $500 \mathrm{MeV}$ (National Bureau of Standards Circular 542, U.S. Government Printing Office, Washington 25, D. C.).

5. P. A. M. Dirac, Approximate Rate of Neutron Multiplication for a Solid of Arbitrary Shape and Uniform Density, British Report M. S. D. 5, Part I. General Theory, 1943.

6. L. Dresner, Resonance Absorption in Nuclear Reactors (Interational Series of Monographs on Nuclear Energy, Vol. 4, Pergamon Press, 1960), p. 65. The Dresner escape probability curves are also presented in R. V. Meghreblian and D. K. Holmes, Reactor Analysis, (McGraw-Hill Book Company, Inc. New York, 1960), p. 671.

7. The sphere curve is clearly the most appropriate for collision calculations related to $\mathrm{Ge}(\mathrm{Li})$ detectors. The infinite cylinder and infinite slab values apply to materials where respectively one or two dimensions are long compared with a collision mean free path-a situation that does not apply here.
8. J. Marshall and A. G. Ward, Can. J. Research A15, 39, (1937).

9. G. Knop and W. Paul, Interaction of Electrons and $\alpha$-Particles with Matter, in Alpha-, Beta-, Gamma-Ray Spectroscopy, Vol. 1, ed. by K. Siegbahn (North-Holland Publishing Co., Amsterdam, 1965), p. 21.

10. This value was calculated directly from the expression given in J. Orear, A. H Rosenfeld, and R. A. Schluter, Nuclear Physics, a course given by Enrico Fermi (University of Chicago Press, 1949), p. 49.

11. J. E. Cline, IEEE Trans. Nucl. Sci. NS-15, No. 3, 198 (1968).

12. K. M. Wainio and G. F. Knoll, Nucl. Instr. Methods 44, 213 (1966).

13. N. V. de Castro Faria and R. J. A. Levesque, Nucl. Instr. Methods 46, 325 (1967).

14. W. Michaelis, Nucl. Instr. Methods 70, 253 (1969).

15. G. V. Walford and C. E. Doust, Nucl. Instr. Methods 67, 272 (1969). 


\section{LEGAL NOTICE}

This report was prepared as an account of Government sponsored work. Neither the United States, nor the Commission, nor any person acting on behalf of the Commission:

A. Makes any warranty or representation, expressed or implied, with respect to the accuracy, completeness, or usefulness of the information contained in this report, or that the use of any information, apparatus, method, or process disclosed in this report may not infringe privately owned rights; or

B. Assumes any liabilities with respect to the use of, or for damages resulting from the use of any information, apparatus, method, or process disclosed in this report.

As used in the above, "person acting on behalf of the Commission" includes any employee or contractor of the Commission, or employee of such contractor, to the extent that such employee or contractor of the Commission, or employee of such contractor prepares, disseminates, or provides access to, any information pursuant to his employment or contract with the Commission, or his employment with such contractor. 
